\title{
Patient-specific blood flow modelling for medical applications
}

\author{
Tatiana Dobroserdova ${ }^{1,2, a}$, Sergey Simakov ${ }^{2,1}$, Timur Gamilov $^{2,1}$, Roman Pryamonosov ${ }^{1}$, and Ekaterina Sakharova ${ }^{3}$ \\ ${ }^{1}$ Institute of Numerical Mathematics of the Russian Academy of Sciences, Gubkin str., 8, Moscow, 119333, Russia \\ ${ }^{2}$ Moscow Institute of Physics and Technology, 9 Institutskiy per., Dolgoprudny, Russia \\ ${ }^{3}$ Higher School of Economics National Research University, 20 Myasnitskaya Ulitsa, Moscow, Russia
}

\begin{abstract}
Patient-specific blood flow modelling helps to predict surgical operation results, gives new noninvasive diagnostic methods, allows to investigate physiology of vascular pathologies. We develop numerical models for such medical applications. 1D blood flow model describes hemodynamics in the whole vascular network. 3D model of fluid flow can be embedded in the 1D model to zoom the domain of interest or to take into account pathologies or implants. We personalize the model, using patient-specific vascular structure and parameters. For some vascular regions extraction of patient-specific geometry can be automatic within developed technology. We verified the 1D model of blood flow. Two examples of real medical applications are presented. The method for FFR estimation is proposed. Also we used the 1D model for prediction of surgical occlusion treatment.
\end{abstract}

\section{Introduction}

Practical medicine has a lot of challenges where mathematical modelling can help significantly. Prediction of surgical operation impact based on numerical simulations (e.g. bypass surgery, stenting) for every certain patient [1] can become real in near future. New noninvasive diagnostic methods, for example estimation of fractional flow reserve [2, 3], are now suggested. New therapeutic methods like enhanced external counterpulsation are being successfully tested with numerical models. Parameters of the patient should be taken into account in the mentioned applications.

Segmentation of patient vascular tree is the basic problem of non-invasive prognostic methodologies. Many researches on blood vessel segmentation were presented and most of them are restricted to one region of interest (cerebral, coronary, abdominal aorta and leg vasculature). Coronary arteries segmentation and centerline extraction algorithms can be completely automated [4]. Techniques for cerebral arteries are often restricted to a braincase, excluding vertebral and carotid arteries, or require user administration. Abdominal aorta region is usually segmented with general methods.

In this paper we discuss out $1 \mathrm{D}$ blood flow model (Section 2). We also mention the possible way to adapt it for a real patient (Section 3). In section 4 we refer to the multiscale 1D-3D modelling as a method to take into account patient-specific pathologies or implants. In section 5 the model is verified. We present two real medical applications of numerical modelling in section 6 .

\section{1D model of global blood circulation}

We consider blood as a viscous incompressible fluid. Every vessel is represented as a tube with elastic walls and the human vascular system is represented by the $1 \mathrm{D}$ graph. Blood flow in every vessel is described by the mass and momentum conservation laws $[5,6]$ :

$$
\begin{gathered}
\frac{\partial S}{\partial t}+\frac{\partial(S u)}{\partial x}=0, \\
\frac{\partial u}{\partial t}+\frac{\partial\left(u^{2} / 2+p / \rho\right)}{\partial x}=\phi,
\end{gathered}
$$

where $S$ is the vessel cross-section area, $u$ is the mean velocity, $p$ is the mean pressure, $\rho$ is the blood density. The term $\phi$ takes into account external forces, such as gravity or friction. The state equation designates elastic properties of the vessel wall and closes the system (1)-(2):

$$
p=\rho c^{2} f(S),
$$

where $c$ is the elasticity model parameter, $f(S)$ is known function [5].

The continuity of solution in vascular anastomoses is prescribed by the enforcing conservation of mass and total pressure continuity. This pair of boundary conditions does not include any additional model parameters and is appropriate for patient-specific modelling. At the same time it was verified for blood flow simulations [7].

For the numerical integration of the model equations (1)-(2), we use the grid-characteristic method [8].

\section{Model initialization}

The 1D model of global blood circulation describes hemodynamics in a 1D vascular network. Length, diameter, elastic parameter should be prescribed to every vessel.

\footnotetext{
a e-mail: DobroserdovaTK@ gmail.com
} 


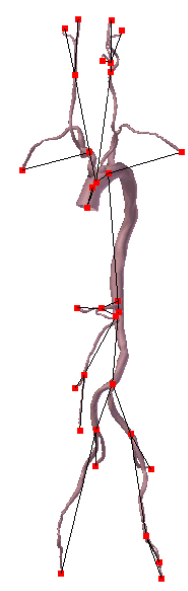

Figure 1. Patient-specific 3D and 1D vascular networks.

\subsection{Reconstruction of the vascular tree}

Patient-specific vascular system is recognized on MRI/CT scans. Segmentation of these scans allows us to reconstruct the 3D geometry of the system (fig. 1).

For some vascular regions extraction of patient specific geometry can be automatic. In case of coronary vessels it requires complex image processing and segmentation algorithms [2] utilizing Hough Circleness transformation and Isoperimetric Distance Trees for aorta initialization. Frangi Vesselness filter is used to find ostia points and segment vessels.

Other regions of circulatory system can be processed in semiautomatic manner. For instance, regions of abdominal aorta and main arteries in legs may be segmented with the tracking technique [1] in the absence of discontinuities, which can be eliminated manually.

Another region of interest is cerebral vasculature. One can take advantage of automatic coronary artery segmentation method [4] if bone intensities are darkened. If only ceCTA (or ceMRA) dataset is given, bone elimination should be done manually. In case when both contrast enhanced and not enhanced datasets are available, bones can be darkened with automatic techniques.

The 3D structure can be transformed into the 1D structure by extracting centerlines everywhere [1]. It is possible to calculate the length of every centerline and the diameter of the $3 \mathrm{D}$ vessel in every point during this algorithm.

\subsection{Parameter initialization}

Every vessel is considered as an elastic tube. Elastic parameters $c$ of the vessel wall can not be measured in vivo with modern methods of diagnostics. It can be retrieved from the literature. The data depends on the individual parameters: age, weight, diseases, profession, smoking and etc. If any patient measurements are available (blood or pulse wave velocities, etc.) model parameters can be fitted to approximate it.

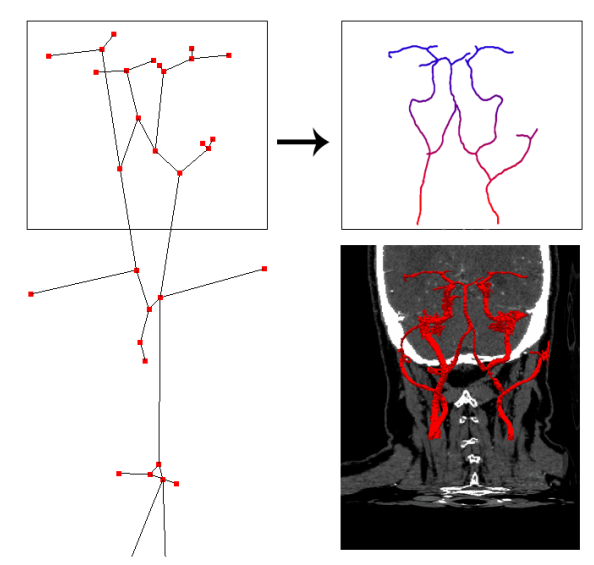

Figure 2. 1D-3D vascular network.

\section{1D-3D modelling}

1D model of global blood circulation describes hemodynamics in healthy vascular system. Medical applications often require blood flow simulations in the vascular network with pathologies or implants. Some pathologies like aneurysm, aorta dissection or placed implants have complex geometry and it is difficult to take them into account by the $1 \mathrm{D}$ model. Often the domain of interest should be considered as 3D. The example of multiscale geometry is presented on the fig.2: cerebral vessels are $3 \mathrm{D}$ and the rest vascular network is $1 \mathrm{D}$.

The 3D model of fluid flow is based on the NavierStokes equations. Vessel walls can be treated as rigid in the $3 \mathrm{D}$ domain. It is the common assumption which helps to save computational time significantly. Also we do not need to tune elastic parameters of the wall which can not be measured for real patient.

Coupling boundary conditions should be specified on the interface between 1D and 3D models. The continuity of normal stress and either continuity of the fluid flux or continuity of linear combination of fluid and energy fluxes can be prescribed. In case of non-physiological reflected waves caused by inconsistency between elasticity effects in the $1 \mathrm{D}$ model and the non-compliance of the 3D model an auxiliary absorbing OD device can be placed at the interface between 1D and 3D models [9]. A virtual device mimics the effect of the 3D model compliance and hence reduces pressure wave reflection and instabilities caused by the inconsistency.

\section{Verification of the 1D blood flow model}

The model of global blood circulation was verified with an experimental model [7]. The experimental model consists of 37 silicone tubes and represents the human arterial system including aorta and carotid arteries. All geometrical and material properties of the arterial network are known. The pulsative pump provides periodic output similar to the aortic flow. Pressure and flow were measured in several 


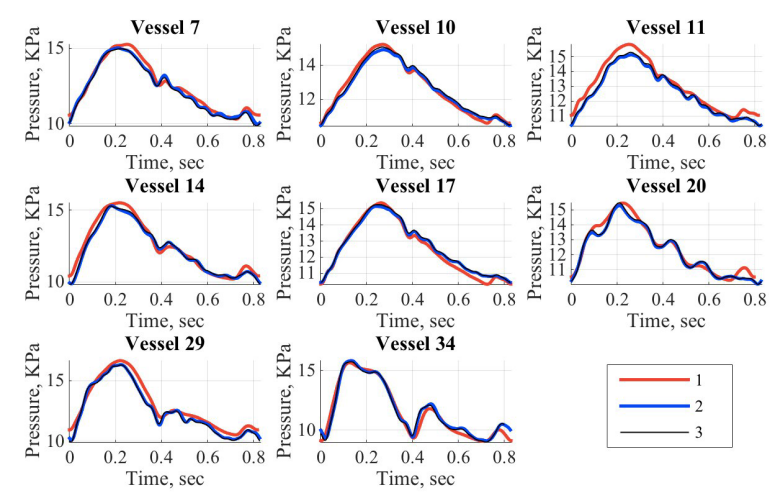

Figure 3. Comparison of pressure in several vessels. Red line (1): experimental measurements; Blue line (2): results of numerical simulation with described model; Black line (3): results of numerical simulation from [7].

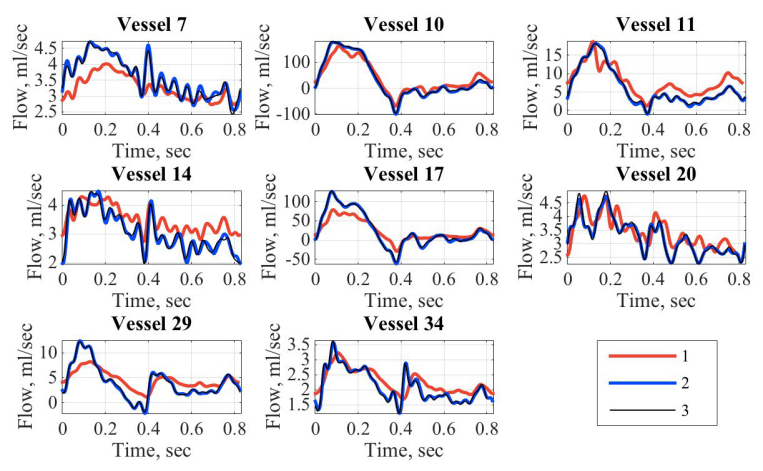

Figure 4. Comparison of fluid flow in several vessels. Red line (1): experimental measurements; Blue line (2): results of numerical simulation with described model; Black line (3): results of numerical simulation from [7].

places during the experiment. The results of the numerical simulation with model described in section 2 (blue line) are presented in fig. 3,4. They well correspond to the experimental data (red line) and to the numerical results of other groups (black line).

Model verification with real human data is rather challenging nowadays. Some parameters of the vascular system can not be measured. Qualitative tests based on patient-specific data were presented before $[1,10]$. At the same time it is difficult to compare quantitative characteristics for such cases.

\section{Medical applications}

The described model can be used for a number of medical applications. Hemodynamic calculations help to assess blood pressure or velocity in various parts of cardiovascular system and predict results of a surgical treatment. Patient-specific data is crucial for such problems. Two examples of real medical applications are presented in the next section.

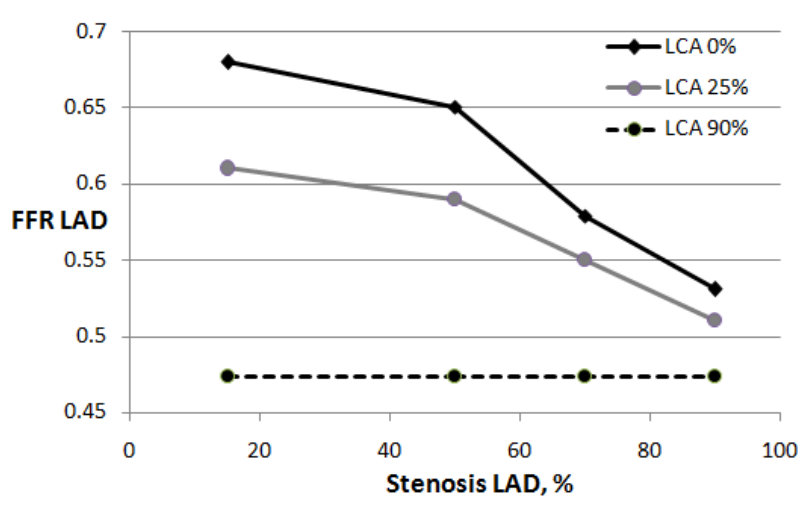

Figure 5. Comparison of FFR in LAD (distal stenosis) for different stenoses in LCA (proximal stenosis) and different stenosis degrees.

\subsection{Fractional flow reserve estimation}

Fractional flow reserve (FFR) measured during invasive coronary angiography is an independent prognosticator for patients with coronary artery diseases and the gold standard for decision making in coronary revascularization. Previously used parameter - percentage of closed lumen (degree of stenosis) - proved to be less accurate [11]. FFR is defined as a ratio between average pressure distal to the stenosis $\left(\bar{P}_{\text {dist }}\right)$ and average aortic pressure $\left(\bar{P}_{a o r}\right)$ in the case of maximum possible hyperemia

$$
F F R=\frac{\bar{P}_{\text {dist }}}{\bar{P}_{\text {aor }}} .
$$

FFR measurement is an expensive and complicated procedure. Hemodynamic simulations can help to estimate FFR without surgical intervention.

Two anonymized patient cases with multiple stenosis of coronary arteries were investigated [2]. The 1D network was constructed on the basis of individual CT-scans. Functional model parameters were assigned to the network with the help of pulse wave velocity studies and other wellknown medical and physiological literature. Personalised virtual FFR was computed. We demonstrated that our approach is capable to predict FFR with acceptable accuracy.

Blood flow model can also be used to study interaction between multiple stenoses and investigate changes in stenosis severety with patient's age. Figure 5 shows how proximal stenosis in left coronary artery (LCA) affects FFR in distal stenosis in left anterior descending artery (LAD).

\subsection{Prediction of surgical atherosclerotic occlusion treatment}

The 1D model of blood flow was used for presurgical patient-specific analysis of the hemodynamic changes due to atherosclerotic occlusion treatment in the femoral artery [1]. The vascular structure of lower extremities was reconstructed from MRI data of the patient. Unmeasured model parameter values (elasticities of vessel walls, resistances for boundary conditions) are fitted to match the 
available presurgical Doppler ultrasound measurements at some points before and after occlusion. These parameters were fitted to reach the best possible correspondance between calculated and measured peak velocities in a set of measurement points. These values also correspond to acceptable ranges for these parameters. Such ranges depend on general physiological and anatomical data, as well as on patient parameters such as height, weight, medical history.

The numerical simulations consider postsurgical blood flow through the left thigh arteries after occlusion treatment. Results of numerical modelling and measured values were compared. We observed quite good coincidence between these data sets: the maximum relative error is not greater than $20 \%$. The error can be caused by the the physiological limitations during parameters fitting procedure and possible input data error.

\section{Conclusions}

In this paper we presented the model of global blood circulation. The described model was verified: the results of numerical experiments correspond to real data. We developed segmentation technology that allows us to get $3 \mathrm{D}$ patient-specific vessel geometry in automatic (for some regions) or semiautomatic manner. The 1D vascular tree can be reconstructed retrieving centerlines from $3 \mathrm{D}$ structure. Parameters were initialized depending on the patient peculiarity. It was used for practical medical applications. Noninvasive fractional flow reserve estimation and prediction of surgical operations are real thanks to numerical simulations.

\section{Acknowledgement}

The results of T.Dobroserdova and R.Pryamonosov presented in section 3.1 and 4 were supported by the Russian Federation President Grant for Young Scientists MK6121.2015.1. Joint author results presented in other sections were supported by the Russian Science Foundation grant 14-31-00024.

\section{References}

[1] T. Gamilov, Yu. Ivanov, P. Kopylov, S. Simakov, Yu. Vassilevski. Patient-specific haemodynamic modeling after occlusion treatment in leg. Mathematical Modelling of Natural Phenomena.,9(6): 85-97 (2014).

[2] T.Gamilov, Ph.Kopylov, R.Pryamonosov, S.Simakov. Virtual fractional flow reserve assesment in patientspecific coronary networks by 1D hemodynamic model. Rus. J. Num. Anal. Math. Mod., 5: 269-276 (2015).

[3] E.Boileau, P.Nithiarasu. One-dimensional modelling of the coronary circulation. Application to noninvasive quantification of fractional flow reserve (FFR). Comp. Exper. Biomed. Sci. Meth. Appl., 21: 137-155 (2015).

[4] Danilov A., Ivanov Y., Pryamonosov R., Vassilevski Y. Methods of graph network reconstruction in personalized medicine. Int J Numer Method Biomed Eng, doi: 10.1002/cnm.2754 (2015).

[5] A. Kholodov, S. Simakov. Computational study of oxygen concentration in human blood under low frequency disturbances. Mathematical models and computer simulations, 1(2):283-295 (2009).

[6] A. Quarteroni, L. Formaggia. Mathematical modelling and numerical simulation of the cardiovascular system. Handb. Numer. Anal., 12: 3-127 (2004).

[7] K.Matthys, J.Alastruey, J.Peiro, A.Khir, P.Segers, P.Verdonck, K.Parker, S.Sherwin. Pulse wave propagation in a model human arterial network: assessment of 1D numerical simulations against in-vitro measurements. J. Biomech., 40(15):3476-3486 (2007).

[8] K.Magomedov, A.Kholodov. Grid-characteristic $\mathrm{Nu}$ merical Methods (Nauka, Moscow, 1988).

[9] T.Dobroserdova, M.Olshanskii, S.Simakov. Multiscale coupling of compliant and rigid walls blood flow models. Int. J. Numer. Meth. Fluids, doi: 10.1002/fld.4241 (2016).

[10] S. Simakov, T. Gamilov, Y.N. Soe. Computational study of blood flow in lower extremities under intense physical load. Russian Journal of Numerical Analysis and Mathematical Modelling., 28(5): 485-504 (2013)

[11] B. De Bruyne, N.Pijls, G.Heyndrickx, D.Hodeige, R.Kirkeeide, K.Gould, Pressure-derived fractional flow reserve to assess serial epicardial stenoses: Theoretical basis and animal validation, Circulation, 15: 1840-1847 (2000). 